\title{
Probing self-interacting dark matter through neutron stars
}

\author{
Yen-Hsun Lin \\ Institute of Physics, Academia Sinica, Taipei 115, Taiwan \\ Department of Physics, National Cheng Kung University, Tainan 701, Taiwan \\ E-mail: yenhsundgate.sinica.edu.tw
}

\begin{abstract}
Compact stellar objects such as neutron stars (NS) are ideal places for capturing dark matter (DM) particles. We study the effect of self-interacting DM captured by the nearby NS that can reheat it to an appreciated surface temperature through absorbing the energy released due to DM annihilation. When DM-nucleon cross section $\sigma_{\chi n}$ is small enough, DM self-interaction will take over the capture process and make the number of captured DM particles increased as well as the DM annihilation rate. The corresponding NS surface temperature resulted from DM self-interaction is about hundreds of Kelvin and is potentially detectable by the future infrared telescopes. Such observations could act as the complementary probe on DM properties to the current DM direct searches.
\end{abstract}

European Physical Society Conference on High Energy Physics - EPS-HEP2019 -

10-17 July, 2019

Ghent, Belgium 


\section{Introduction}

A compact stellar object such as neutron star (NS) is a perfect place to capture DM particles even when DM-nucleon cross section $\sigma_{\chi_{n}}$ is way smaller than the current direct search limits. Investigations on DM in compact stellar objects are studied recently in Refs. [1, 2, 3, 4, 5, 6, 7, 8, $10,9,11,12]$. Due to strong gravitational field, DM evaporation mass for NS is less than $10 \mathrm{keV}$ [6]. Therefore, NS is sensitive to a broad spectrum of DM mass from $10 \mathrm{keV}$ to PeV, sometimes it can be even extended to higher mass region. Unlike the Sun, it loses its sensitivity to DM when $m_{\chi} \lesssim 5 \mathrm{GeV}$ as a consequence of evaporation [13, 14]. In the later discussion, we will focus on the Weakly Interacting Massive Particle (WIMP) scenario with mass from $\mathrm{MeV}$ to hundreds of GeVs.

An old NS having age greater than billions of years could become a cold star after processing several cooling mechanism by emitting photons and neutrinos [15, 16]. However, if the residing DM particles in the NS can annihilate to SM particles other than neutrinos, they will be absorbed by the host star and act like energy injections to heat the star up [1, 2, 3]. In addition, recent literature also suggests that the halo DM particles constantly bombard NS can deposit their kinetic energy to the star. This is called dark kinetic heating [17]. These two contributions might prevent NS from inevitable cooling as suggested by Refs. [17, 18].

Besides the DM-nucleon interaction, inconsistencies in the small-scale structure between the observations and the $N$-body simulations $[19,20,21]$ imply the existence of self-interacting DM (SIDM) [22, 23]. The constraint given in Ref. [24, 25] could mitigate such discrepancies as well as the diversity problem of the galactic rotation curves $[26,27]$. It brings us to

$$
3 \mathrm{~cm}^{2} \mathrm{~g}^{-1} \leq \sigma_{\chi \chi} / m_{\chi} \leq 6 \mathrm{~cm}^{2} \mathrm{~g}^{-1}
$$

where $\sigma_{\chi \chi}$ is DM self-interaction cross section. The resulting effect of DM self-capture in NS was considered insignificantly due to it saturates quickly when the sum of the individual $\sigma_{\chi \chi}$ exceeds the geometrical area over which DM is thermally distributed [6]. Its impact is unable to compete with the capture by DM-nucleon interaction when $\sigma_{\chi_{n}} \gtrsim 10^{-50} \mathrm{~cm}^{2}$. However, current direct searches have put more stringent limits on $\sigma_{\chi n}$ to test. If it is small enough, DM self-capture will eventually take over [7]. In this region, DM self-interaction will re-enhance the captured DM particles as well as the DM annihilation rate regardless how small $\sigma_{\chi_{n}}$ is. The corresponding energy injection increases consequently. Hence, in the self-interaction dominant region, NS will experience a reheating effect with rising NS surface temperature [3].

\section{DM captured by NS}

When the halo DM particles scatter with NS and lose significant amount of energies, they will be gravitationally bounded in the star. The evolution of DM number $N_{\chi}$ in NS can be characterized by the differential equation

$$
\frac{d N_{\chi}}{d t}=C_{c}+C_{s} N_{\chi}-C_{a} N_{\chi}^{2}
$$

where $C_{c}$ is the capture rate due to DM-nucleon interaction, $C_{s}$ the DM self-capture rate due to DM self-interaction and $C_{a}$ the DM annihilation rate. A general solution to $N_{\chi}$ is given by

$$
N_{\chi}(t)=\frac{C_{c} \tanh (t / \tau)}{\tau^{-1}-C_{s} \tanh (t / \tau) / 2}
$$


where $\tau=1 / \sqrt{C_{c} C_{a}+C_{s}^{2} / 4}$ is the equilibrium timescale. In the case of $t \gg \tau, d N_{\chi} / d t=0$ where $N_{\chi}$ reaches the steady state. Hence we have

$$
N_{\chi}(t \gg \tau) \equiv N_{\chi, \mathrm{eq}}=\sqrt{\frac{C_{c}}{C_{a}}}\left(\sqrt{\frac{R}{4}}+\sqrt{\frac{R}{4}+1}\right)
$$

where

$$
R \equiv \frac{C_{s}^{2}}{C_{c} C_{a}} \begin{cases}\gg 1, & C_{s} \text {-dominant } \\ \ll 1, & C_{c} \text {-dominant }\end{cases}
$$

Thus, $R$ signifies how crucial that the DM self-capture is in the DM evolution in NS. Additionally, we can obtain two solutions to $N_{\chi}$ when $d N_{\chi} / d t=0$, by examining Eq. (2.1),

$$
N_{\chi, \mathrm{eq}}^{R \ll 1}=\sqrt{\frac{C_{c}}{C_{a}}} \quad \text { and } \quad N_{\chi, \mathrm{eq}}^{R \gg 1}=\frac{C_{s}}{C_{a}} .
$$

That means, either the capture is dominated by $C_{c}$ or $C_{s}$ that could accumulate the same amount of DM particles in NS.

\section{NS cooling and DM energy injection}

After the birth of NS, it undergoes the cooling mechanism due to neutrino and photon emissions $[15,16]$. Nonetheless, if the residing DM particles in NS can annihilate, the annihilation products will be absorbed and act like energy injections to heat the host star up. The NS interior temperature $T_{\text {int }}$ can be described by the following differential equation

$$
\frac{d T_{\text {int }}}{d t}=\frac{-\varepsilon_{v}-\varepsilon_{\gamma}+\varepsilon_{\chi}}{c_{V}}
$$

where $\varepsilon_{v, \gamma, \chi}$ are the emissivities due to neutrino emission, photon emission and DM respectively and $c_{V}$ the NS heat capacity of NS .

Since the NS outer envelope shields us from observing $T_{\text {int }}$ directly. We can only observe the luminosity $L_{\gamma}$ emitted from this envelope. The corresponding temperature can be inferred from Stefan-Boltzmann's law that is defined as the NS surface temperature $T_{\text {sur }}$ where $L_{\gamma}=4 \pi R^{2} \sigma_{\mathrm{SB}} T_{\text {sur }}^{4}$. A relation that connects $T_{\text {int }}$ and $T_{\text {sur }}$ is given by $[16,28,29]$

$$
T_{\text {sur }}=0.87 \times 10^{6} \mathrm{~K}\left(\frac{g_{s}}{10^{14} \mathrm{~cm} \mathrm{~s}^{-2}}\right)^{1 / 4}\left(\frac{T_{\text {int }}}{10^{8} \mathrm{~K}}\right)^{0.55}
$$

where $g_{s}=G M / R^{2}=1.85 \times 10^{14} \mathrm{~cm} \mathrm{~s}^{-2}$ is the surface gravity. In general, $T_{\text {sur }}$ is lower than $T_{\mathrm{int}}$. However, when $T_{\text {int }} \lesssim 3700 \mathrm{~K}$, the distinction between the two becomes negligible [16]. Applying Eq. (3.2) to obtain $T_{\text {sur }}$ from $T_{\text {int }}$ is unnecessary when $T_{\text {int }} \lesssim 3700 \mathrm{~K}$.

On the other hand, $L_{\gamma}$ is also responsible for the energy loss due to photon emission. Hence we have the effective photon emissivity [3]

$$
\varepsilon_{\gamma}=\frac{L_{\gamma}}{(4 / 3) \pi R^{3}} \approx \begin{cases}2.71 \times 10^{-17} \mathrm{GeV}^{4} \mathrm{yr}^{-1}\left(\frac{T_{\text {int }}}{10^{8} \mathrm{~K}}\right)^{2.2} & T_{\mathrm{int}} \gtrsim 3700 \mathrm{~K}, \\ 2.56 \times 10^{-9} \mathrm{GeV}^{4} \mathrm{yr}^{-1}\left(\frac{T_{\mathrm{int}}}{10^{8} \mathrm{~K}}\right)^{4} & T_{\mathrm{int}} \lesssim 3700 \mathrm{~K},\end{cases}
$$


where Eq. (3.3a) is obtained from Ref. [1] and Eq. (3.3b) is the expression for $\varepsilon_{\gamma}$ when $T_{\text {int }} \lesssim$ $3700 \mathrm{~K}$.

In addition, NS heating comes from the contributions of DM annihilation and dark kinetic heating. They are given by

$$
\mathscr{E}_{\chi}=2 m_{\chi} \Gamma_{A}=m_{\chi} C_{a} N_{\chi}^{2} f_{\chi}
$$

for annihilation and

$$
\mathscr{K}_{\chi}=C_{c} E_{s}
$$

for dark kinetic heating. The factor $f_{\chi}$ characterizes the energy absorption efficiency which runs from 0 to 1 . The term $E_{s}=m_{\chi}(\gamma-1)$ is the DM kinetic energy deposited in NS and $\gamma \simeq 1.35$ [17]. Therefore, we have

$$
\varepsilon_{\chi}=\frac{\mathscr{E}_{\chi}+\mathscr{K}_{\chi}}{4 \pi R^{3} / 3}
$$

for DM emissivity.

\section{Numerical results}

Here we display our final result in Fig. 1 as well as the constraints on $\sigma_{\chi^{n}}$ from different DM direct searches. The purple shaded region is where DM heating has no contribution. Thus, standard NS cooling mechanism predicts $T_{\text {sur }} \approx 120 \mathrm{~K}$ for an isolated two-billion-year old NS. Above the shaded region, the larger $\sigma_{\chi n}$, the more $N_{\chi}$ will be captured as well as the stronger DM heating from annihilation. Below the shaded region, $T_{\text {sur }}$ is reheated as a consequence of DM self-interaction and the color portion is the maximum $T_{\text {sur }}$ can be obtained in the DM self-interaction dominant region.

Interestingly, $T_{\text {sur }}$ above the purple shaded region coincides with $T_{\text {sur }}$ in some parameter space below the purple shaded region. This phenomenon is indicated in Eq. (2.5). For instance, there are two lines show $T_{\text {sur }}=300 \mathrm{~K}$ in Fig. 1 . It could be helpful as an extra information for determining DM properties along with current DM direct searches. If we observed $T_{\text {sur }}=300 \mathrm{~K}$ for an isolated two-billion-year old NS and concur that the heating is purely from DM. But $10 \mathrm{GeV} \lesssim m_{\chi} \lesssim$ $100 \mathrm{GeV}$ with $T_{\text {sur }}=300 \mathrm{~K}$ is already disfavored by DARWIN. Thus, DM could be either lighter than a few $\mathrm{GeV}$ or $\sigma_{\chi n}$ is very small. Future DM direct searches could reveal more constraint on sub-GeV DM, together with the astrophysical observations on $T_{\text {sur }}$, we can further unravel more information about DM.

\section{Summary}

In this work, we found that when DM self-interaction dominates DM capture process, $T_{\text {sur }}$ increases as $\sigma_{\chi n}$ decreases. This is contrary to DM-nucleon interaction dominant case where $T_{\text {sur }}$ becomes colder as $\sigma_{\chi^{n}}$ diminishes.

In addition, when $10^{-50} \mathrm{~cm}^{2} \lesssim \sigma_{\chi n} \lesssim 10^{-57} \mathrm{~cm}^{2}$, the heating from DM is negligible. The energy loss from photon emission overwhelms the energy deposition from DM annihilation. Thus, standard NS cooling is the dominant process. For a two-billion-year old isolated NS, standard NS cooling predicts a lower bound $T_{\text {sur }} \approx 120 \mathrm{~K}$. If DM properties such as $m_{\chi}$ and $\sigma_{\chi n}$ lie within this parameter space, they are unable to probe from the measurement of $T_{\text {sur. }}$. However, the precise 


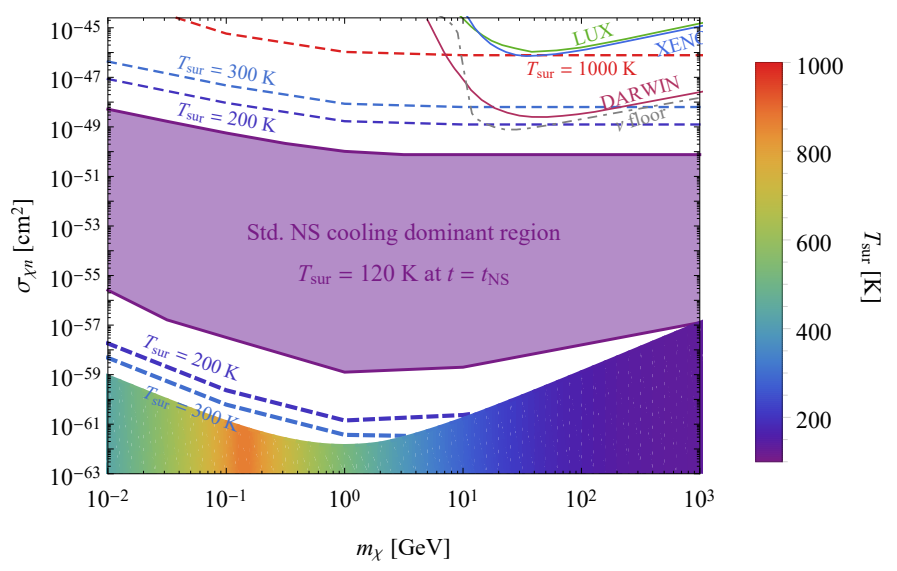

Figure 1: The purple shaded area is where standard NS cooling overwhelms the DM heating. The corresponding $T_{\text {sur }}$ is about $120 \mathrm{~K}$. DM-nucleon interaction and DM self-interaction are responsible for the heating on $T_{\text {sur }}$ above and below the purple shaded region in the middle of this figure respectively. We use $\sigma_{\chi \chi} / m_{\chi}=4 \mathrm{~cm}^{2} \mathrm{~g}^{-1}$ and $\langle\sigma v\rangle=3 \times 10^{-26} \mathrm{~cm}^{3} \mathrm{~s}^{-1}$ in the calculation. See main text for detail. The constraints on $\sigma_{\chi n}$ from different DM direct searches such as DARWIN [?], LUX [?] and XENON1T [?] are shown in the plot as well.

value for such lower bound depends on the knowledge of NS cooling mechanism. Once we have more constraints from the astrophysical observations on $T_{\text {sur }}$ for isolated old NSs, this lower bound could be subject to some correction.

The parameter space in the capture processes dominated by DM-nucleon interaction and by DM self-interaction can generate the same $T_{\text {sur }}$ as shown in Fig. 1. Together with the current DM direct searches, it could improve our knowledge on DM properties in various ways.

In closing, the NS surface temperature $T_{\text {sur }}$ induced by DM self-interaction roughly ranges from $120 \mathrm{~K}$ to $700 \mathrm{~K}$. The corresponding blackbody peak wavelength is infrared and could be detected by the forthcoming telescopes such as JWST, TMT and E-ELT. The corresponding observations on $T_{\text {sur }}$ could act as the complementary probe to DM direct searches in the future.

\section{References}

[1] C. Kouvaris, Phys. Rev. D 77, 023006 (2008) [arXiv:0708.2362 [astro-ph]].

[2] A. de Lavallaz and M. Fairbairn, Phys. Rev. D 81, 123521 (2010) [arXiv:1004.0629 [astro-ph.GA]].

[3] C. S. Chen and Y. H. Lin, JHEP 1808, 069 (2018) [arXiv:1804.03409 [hep-ph]].

[4] C. Kouvaris and P. Tinyakov, Phys. Rev. D 82, 063531 (2010) [arXiv:1004.0586 [astro-ph.GA]].

[5] C. Kouvaris and P. Tinyakov, Phys. Rev. D 83, 083512 (2011) [arXiv:1012.2039 [astro-ph.HE]].

[6] S. D. McDermott, H. B. Yu and K. M. Zurek, Phys. Rev. D 85, 023519 (2012) [arXiv:1103.5472 [hep-ph]].

[7] T. Güver, A. E. Erkoca, M. Hall Reno and I. Sarcevic, JCAP 1405, 013 (2014) [arXiv:1201.2400 [hep-ph]].

[8] J. Bramante, K. Fukushima, J. Kumar and E. Stopnitzky, Phys. Rev. D 89, 015010 (2014) [arXiv:1310.3509 [hep-ph]]. 
[9] L. Tolos and J. Schaffner-Bielich, Phys. Rev. D 92, 123002 (2015) [arXiv:1507.08197 [astro-ph.HE]].

[10] J. Bramante, A. Delgado and A. Martin, Phys. Rev. D 96, 063002 (2017) [arXiv:1703.04043 [hep-ph]].

[11] J. Ellis, A. Hektor, G. Hütsi, K. Kannike, L. Marzola, M. Raidal and V. Vaskonen, arXiv:1710.05540 [astro-ph.CO].

[12] J. Ellis, G. Hütsi, K. Kannike, L. Marzola, M. Raidal and V. Vaskonen, arXiv:1804.01418 [astro-ph.CO].

[13] A. Gould, Astrophys. J. 321, 560 (1987).

[14] C. S. Chen, F. F. Lee, G. L. Lin and Y. H. Lin, JCAP 1410, 049 (2014) [arXiv:1408.5471 [hep-ph]].

[15] S. L. Shapiro and S. A. Teukolsky, Black holes, white dwarfs, and neutron stars: The physics of compact objects, New York, USA: Wiley (1983) 645 p

[16] D. Page, J. M. Lattimer, M. Prakash and A. W. Steiner, Astrophys. J. Suppl. 155, 623 (2004) [astro-ph/0403657].

[17] M. Baryakhtar, J. Bramante, S. W. Li, T. Linden and N. Raj, Phys. Rev. Lett. 119, 131801 (2017) [arXiv:1704.01577 [hep-ph]].

[18] N. Raj, P. Tanedo and H. B. Yu, Phys. Rev. D 97, 043006 (2018) [arXiv:1707.09442 [hep-ph]].

[19] J. F. Navarro, C. S. Frenk and S. D. M. White, Astrophys. J. 490, 493 (1997) [astro-ph/9611107].

[20] B. Moore, Nature 370, 629 (1994).

[21] R. A. Flores and J. R. Primack, Astrophys. J. 427, L1 (1994) [astro-ph/9402004].

[22] D. N. Spergel and P. J. Steinhardt, Phys. Rev. Lett. 84, 3760 (2000) [astro-ph/9909386].

[23] S. Tulin and H. B. Yu, Phys. Rept. 730, 1 (2018) [arXiv:1705.02358 [hep-ph]].

[24] A. Kamada, M. Kaplinghat, A. B. Pace and H. B. Yu, Phys. Rev. Lett. 119, 111102 (2017) [arXiv:1611.02716 [astro-ph.GA]].

[25] A. Robertson et al., arXiv:1711.09096 [astro-ph.CO].

[26] K. A. Oman et al., Mon. Not. Roy. Astron. Soc. 452, 3650 (2015) [arXiv:1504.01437 [astro-ph.GA]].

[27] O. D. Elbert, J. S. Bullock, M. Kaplinghat, S. Garrison-Kimmel, A. S. Graus and M. Rocha, Astrophys. J. 853, 109 (2018) [arXiv:1609.08626 [astro-ph.GA]].

[28] E. H. Gudmunsson, C. J. Pethick and R. I. Epstein, Astrophys. J. 259, L19, (1982)

[29] E. H. Gudmunsson, C. J. Pethick and R. I. Epstein, Astrophys. J. 272, 286, (1983)

[30] B. Bertoni, A. E. Nelson and S. Reddy, Phys. Rev. D 88, 123505 (2013) [arXiv:1309.1721 [hep-ph]]. 